\title{
Pannus-related left main trunk ostial stenosis after aortic valve replacement
}

\author{
Taiyo Jinno $^{1}$, Yasuyuki Kato ${ }^{2}$, Hidetaka Yamauchi ${ }^{1}$, Yusuke Date ${ }^{1}$, Kenichi Sasaki ${ }^{1}$, \\ Atsushi Shimizu ${ }^{3}$, Hiroshi Kiyama ${ }^{1}$, and Toshiya Koyanagi ${ }^{1}$ \\ ${ }^{1}$ Affiliation not available \\ ${ }^{2}$ Osaka City General Hospital \\ ${ }^{3}$ Saitama Seikeikai Hospital
}

March 20, 2021

\begin{abstract}
A 34-year-old woman was hospitalized with shortness of breath and chest tightness and pain. She had undergone aortic valve replacement for aortic stenosis at the age of 18 years. Transthoracic echocardiography showed left ventricular asynergy and a high aortic valve pressure gradient. Thus, structural valve deterioration was diagnosed. Coronary computed tomography and coronary angiography revealed left main trunk ostial stenosis that had caused acute anteroseptal myocardial infarction. Urgent surgery revealed pannus formation around the prosthetic valve and covering the ostium of the left main trunk. A Bentall procedure and coronary artery bypass grafting were performed. The postoperative course was uneventful.
\end{abstract}

\section{Hosted file}

JCS manuscript \begin\{CJK\}\{UTF8\}\{gbsn\}. \end\{CJK\}\selectlanguage\{english\}pdf available at } https://authorea. com/users/402804/articles/514553-pannus-related-left-main-trunk-ostialstenosis-after-aortic-valve-replacement 


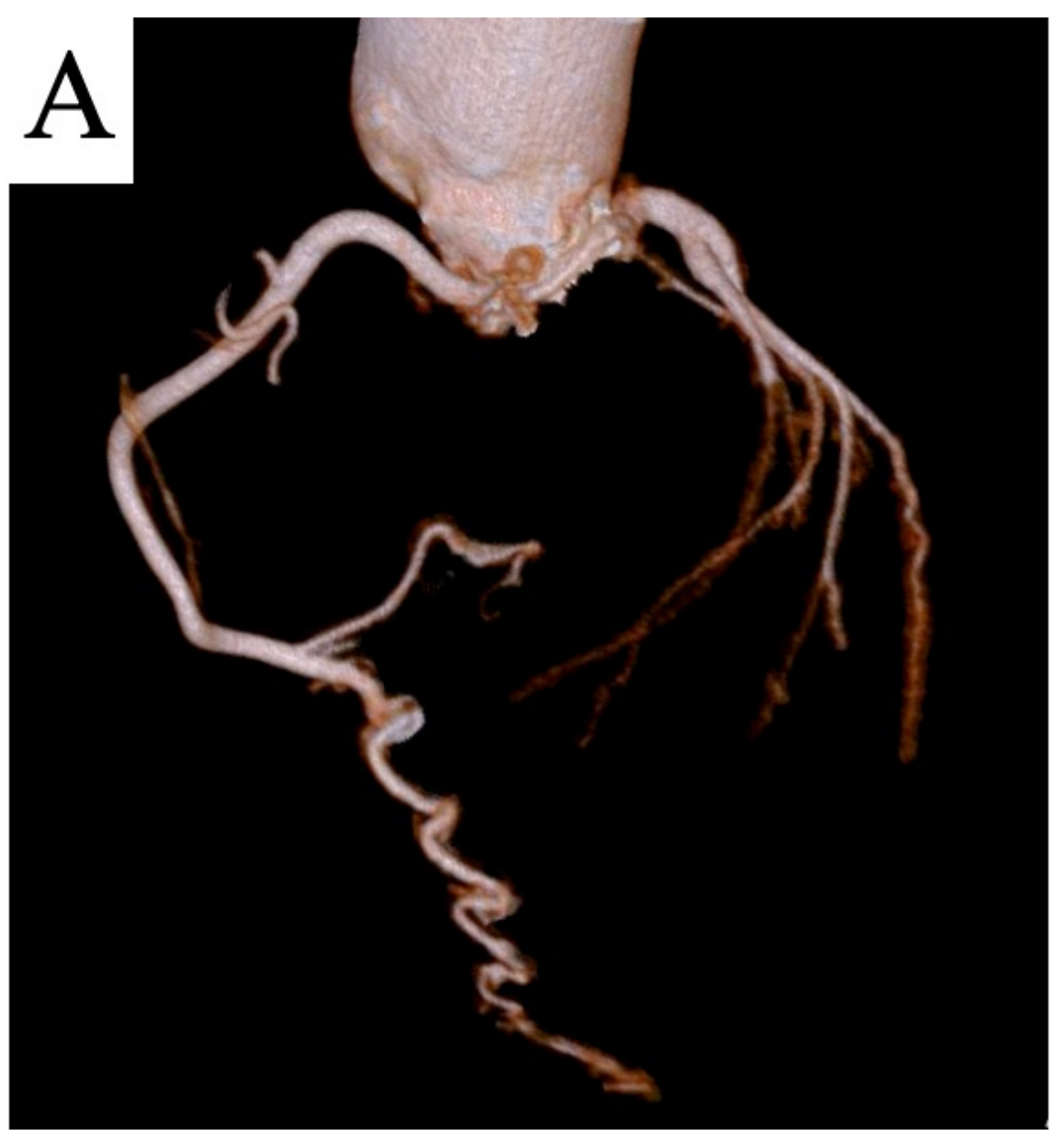




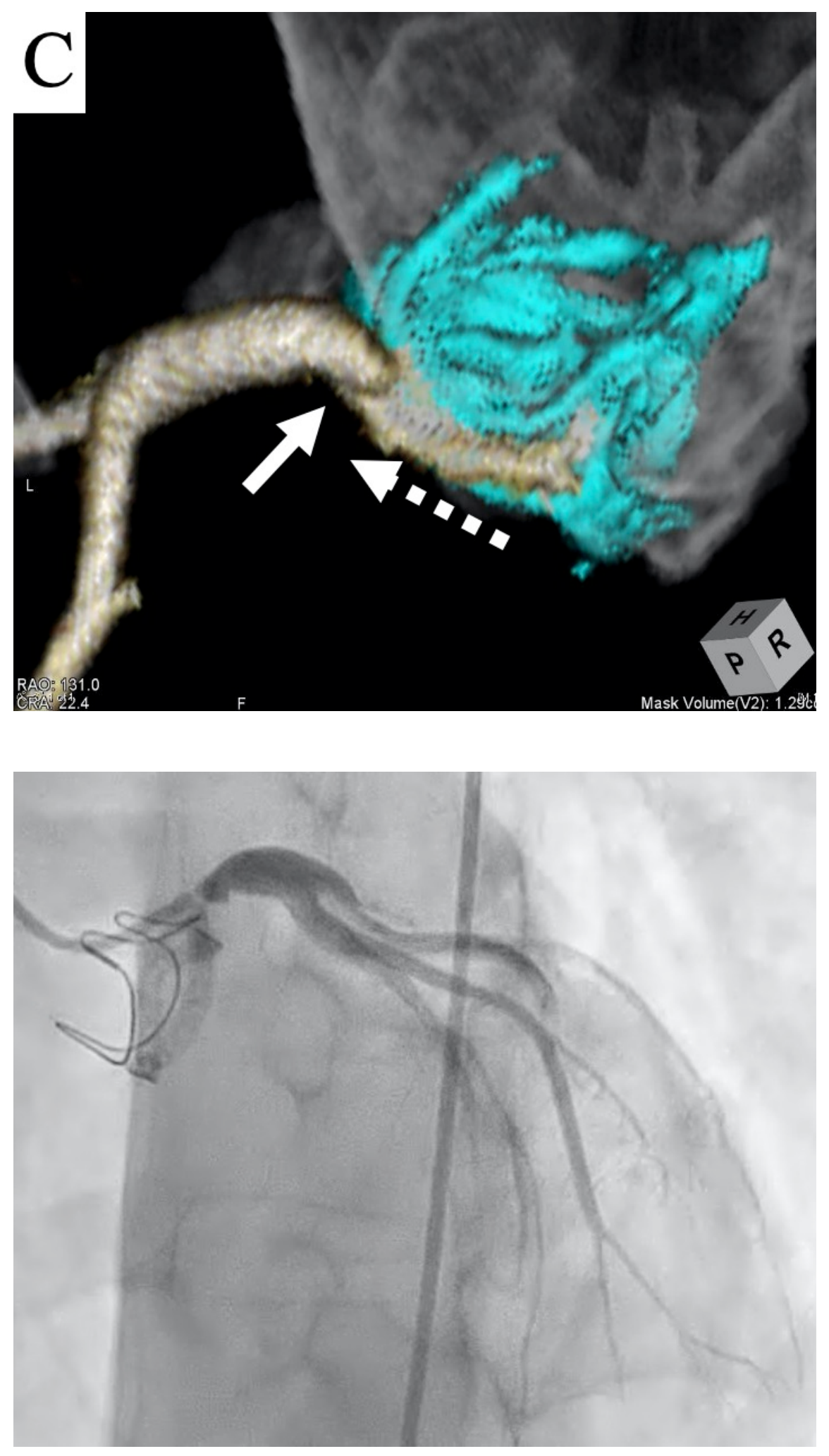

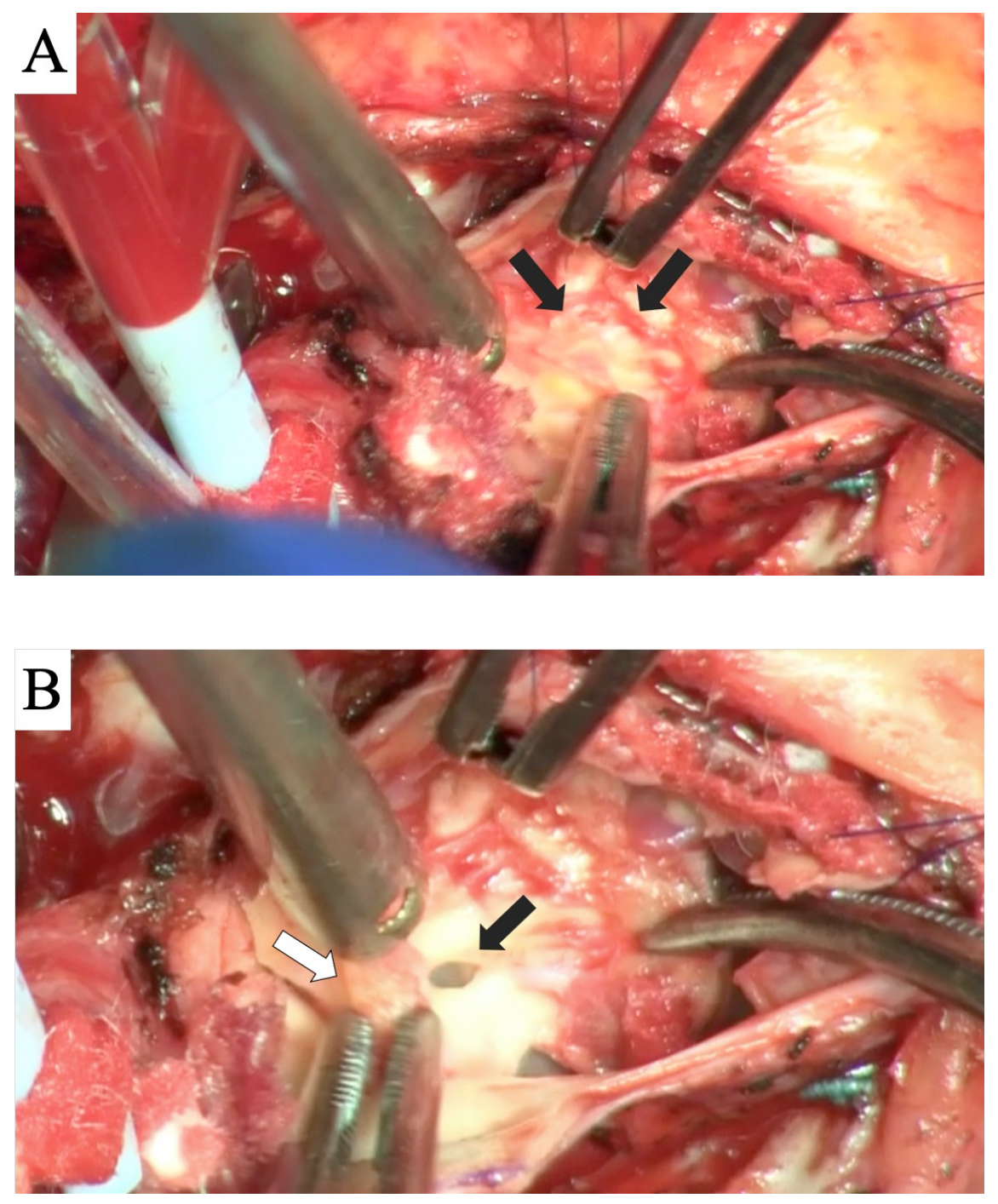